\title{
Informações sobre a episiotomia recebidas pelas mulheres durante o processo de parto e nascimento
}

\author{
Information on episiotomy received by women during labor and delivery \\ Información sobre la episiotomía recibida por mujeres durante el trabajo de parto y el \\ parto
}

Bruna de Oliveira Jochims ${ }^{1 *}$, Cláudia Junqueira Armellini ${ }^{1}$, Helga Geremias Gouveia ${ }^{1}$.

\section{RESUMO}

Objetivo: Identificar o conhecimento e as informações recebidas sobre a episiotomia pelas mulheres durante o processo de parto e nascimento. Método: Trata-se de um estudo quantitativo de corte transversal, realizado em um hospital terciário, público e universitário. A amostra foi composta por puérperas submetidas à episiotomia. A coleta de dados ocorreu no período de fevereiro a setembro de 2016. Os dados foram submetidos a análise descritiva por meio de frequências absolutas e relativas. Resultados: Participaram do estudo, 246 puérperas. Verificou-se que $96,3 \%(n=237)$ das mulheres conheciam as condições de seu períneo no pós-parto, $77,2 \%(n=190)$ referiram ter recebido informações de que seriam submetidas à episiotomia previamente a realização do procedimento, $65,0 \%(n=160)$ foram informadas sobre o motivo da realização da episiotomia e $75,0 \%$ dos motivos de episiotomia relatados pelas mulheres foram "para aumentar a passagem para o bebê". Conclusão: A maioria das mulheres conhecia as condições de seu períneo, foi informada que seria submetida à episiotomia e recebeu informação sobre o motivo desse procedimento. Apesar de ser considerada um procedimento cirúrgico e que necessita de consentimento para sua realização, houve mulheres expostas a episiotomia sem informações suficientes acerca da mesma.

Palavras-chave: Episiotomia, Direitos do Paciente, Informação, Enfermagem Obstétrica.

\begin{abstract}
Objective: To identify the knowledge and information received about episiotomy by women during the labor and delivery process. Method: This is a cross-sectional quantitative study, performed in a tertiary, public and university hospital. The sample consisted of postpartum women submitted to episiotomy. Data were collected from February to September 2016. Data were submitted to descriptive analysis using absolute and relative frequencies. Results: 246 postpartum women participated in the study. It was verified that $96.3 \%(n=237)$ of the women knew the conditions of their postpartum perineum, $77.2 \%(n=190)$ reported receiving information that they would undergo the episiotomy prior to performing the procedure, $65.0 \%(n=160)$ were informed about the reason for the episiotomy and $75.0 \%$ of the episiotomy reasons reported by the women were "to increase the passage to the baby." Conclusion: Most of the women knew the conditions of their perineum, were informed that they would be submitted to episiotomy and received information about the reason for this procedure. Despite being considered a surgical procedure and requiring consent for its accomplishment, there were women exposed to episiotomy without sufficient information about the same.
\end{abstract}

Key words: Episiotomy, Patient Rights, Information, Obstetric Nursing.

${ }^{1}$ Universidade Federal do Rio Grande do Sul (UFRGS), Porto Alegre-RS.`E-mail: brujochims@hotmail.com

SUBMETIDO EM: 7/2019

ACEITO EM: 8/2019

PUBLICADO EM: 9/2019 


\section{RESUMEN}

Objetivo: Identificar el conocimiento y la información recibida acerca de la episiotomía realizada por mujeres durante el trabajo de parto y el parto. Método: Este es un estudio cuantitativo de corte transversal, realizado en un hospital terciario, público y universitario. La muestra consistió en mujeres postparto sometidas a episiotomía. Los datos se recopilaron de febrero a septiembre de 2016. Los datos se enviaron a un análisis descriptivo utilizando frecuencias absolutas y relativas. Resultados: 246 mujeres en posparto participaron en el estudio. Se verificó que el $96,3 \%(n=237)$ de las mujeres conocía las condiciones de su perineo posparto, el $77,2 \%(n=190)$ informó haber recibido información de que se sometería a la episiotomía antes de realizar el procedimiento, $65.0 \%(n=160)$ fueron informados sobre la razón de la episiotomía y el $75.0 \%$ de las razones de episiotomía reportadas por las mujeres fueron "para aumentar el paso al bebé". Conclusión: la mayoría de las mujeres conocían las condiciones de su perineo, se les informó que serían sometidas a una episiotomía y recibieron información sobre el motivo de este procedimiento. A pesar de ser considerado un procedimiento quirúrgico y requerir el consentimiento para su realización, hubo mujeres expuestas a la episiotomía sin información suficiente al respecto.

Palabras clave: Episiotomía, Derechos del Paciente, Información, Enfermería Obstétrica.

\section{INTRODUÇÃO}

O parto é um evento único para cada mulher e todos que dele participam, sendo um processo natural e fisiológico (ANDRADE MAC e LIMA JBMC, 2014). Porém, atualmente, o parto passou a ser considerado um evento patológico, tratando a mulher como incapaz de parir de forma segura e satisfatória (ANDRADE BP e LIMA JBMC, 2014). As intervenções passam a ter conotação de proteção e cuidado, deixando de ser utilizadas somente para prevenção de desfechos desfavoráveis (RIESCO MLG, 2014).

Dentre essas intervenções encontra-se a episiotomia, um corte no períneo que atinge pele, músculos, tendões e vasos sanguíneos, feito com tesoura ou bisturi, com o intuito de ampliar o canal de nascimento (JIANG H, et al., 2017). Atualmente, não há evidências científicas que definam os critérios exatos para indicação da episiotomia (JIANG H, et al., 2017; WORLD HEALTH ORGANIZATION, 2018).

Embora seja considerado um procedimento cirúrgico, com justificativa e descrição registradas em prontuário da paciente no Brasil ainda ocorre da episiotomia ser realizada sem o profissional informar previamente à mulher sobre sua necessidade e seus riscos, e sem obter o seu consentimento (MARTINSCOSTA SH, et al., 2017; BOLSONI AC e COELHO JA, 2016; MEDEIROS NCM, et al., 2016).

Percebe-se que há déficit de conhecimento das mulheres sobre a nomenclatura, as indicações e a realização da episiotomia, havendo também influência cultural sobre as crenças acerca desta prática, sendo associada, pelas mulheres, a um procedimento necessário e benéfico para a mãe e bebê (POMPEU KC, et al., 2017).

Portanto, atos como não informar, não adquirir consentimento da parturiente sobre essas intervenções ou realizar procedimentos com fins didáticos correspondem a uma forma de violência obstétrica, todavia, esta é dificilmente reconhecida devido ao momento em que é imposta, pois a mulher se encontra mais vulnerável devido ao processo de parturição (ANDRADE BP e AGGIO CM, 2014).

A episiotomia é uma das formas de violência obstétrica mais praticada e nota-se uma tendência de minimizá-la, tanto pelo profissional que a pratica quanto pela mulher que possui pouca informação acerca do procedimento, ao referirem-se a mesma como um "cortezinho", a fim de torná-la insignificante (ROCHA MJ e GRISI EP, 2017; POMPEU KC, et al., 2017).

Diante da importância do conhecimento das mulheres sobre a assistência prestada durante o parto e da necessidade de consentimento da mulher perante os procedimentos realizados durante esse processo, destaca-se a relevância da temática deste estudo. Acredita-se que os resultados poderão contribuir para a reflexão e avaliação sobre as práticas assistenciais dos profissionais que atuam durante o parto e nascimento, 
inclusive do enfermeiro, profissional presente em todas as etapas do processo de parturição, e também contribuir para o desenvolvimento de estratégias assistenciais com o intuito de melhorias nas taxas de episiotomia e informações às mulheres acerca dessa prática.

Sendo assim, o objetivo desse estudo foi identificar o conhecimento e as informações recebidas sobre a episiotomia pelas mulheres durante o processo de parto e nascimento.

\section{MÉTODOS}

Trata-se de estudo quantitativo de corte transversal, onde foi realizada uma subanálise da pesquisa intitulada "Práticas de atendimento implementadas durante o processo de parturição", desenvolvida em Unidade de Internação Obstétrica do Hospital de Clínicas de Porto Alegre (HCPA), onde a assistência ao parto é prestada por médicos obstetras, residentes de obstetrícia e acadêmicos de medicina. Para o cálculo do tamanho amostral daquele estudo foi utilizado o programa Winpepi, versão 11.43 , considerando poder de $80 \%$, nível de significância de $5 \%$, baseado em dados institucionais sobre boas práticas na atenção e diferença entre as proporções dos desfechos do recém-nascido cujo Odds Ratio (OR) seja de 0.6 (VOGT SE, et al., 2014). Chegou-se ao tamanho da amostra de 586 puérperas, sendo incluídas mulheres que tiveram parto na Unidade de Centro Obstétrico (UCO) da instituição e recém-nascidos com idade gestacional maior ou igual a 37 semanas, calculada pelo método de Capurro. Foram excluídas puérperas com indicação eletiva de cesariana, parto gemelar, óbito fetal, malformação fetal e internadas via custeio próprio e via prestadores de saúde privada.

Os dados foram coletados no período de fevereiro a setembro de 2016, utilizando-se registros em prontuário, Planilha de Ocorrências da UCO e questionário estruturado, aplicado às puérperas com o mínimo de 12 horas pós-parto.

Para o presente estudo, foram incluídas todas as mulheres submetidas à episiotomia. Utilizaram-se variáveis referentes à caracterização da amostra (sociodemográficas e obstétricas), ao conhecimento das mulheres sobre as condições do seu períneo após o parto, à informação prévia recebida de que seria submetida à episiotomia, à informação sobre o motivo da episiotomia e ao conhecimento da mulher sobre esse motivo. Os dados foram submetidos à análise descritiva por meio de frequências absolutas e relativas com o emprego do software SPSS versão 18.

O projeto do qual esse estudo deriva foi apreciado pela Comissão de Pesquisa da Escola de Enfermagem da Universidade Federal do Rio Grande do Sul e pelo Comitê de Ética em Pesquisa do Grupo de Pesquisa e Pós-graduação do HCPA (no 1.394.513). Foram cumpridos os termos da Resolução no 466/2012 do Conselho Nacional de Saúde. Todas as participantes ou seu responsável, no caso de menores de 18 anos, assinaram o Termo de Consentimento Livre e Esclarecido. Foi apresentado à Instituição o Termo de Responsabilidade para Utilização de Dados Institucionais.

\section{RESULTADOS}

Amostra deste estudo foi composta por todas mulheres submetidas ao parto vaginal com episiotomia, num total de 246 , sendo que $10,6 \%(n=26)$ tiveram parto com uso de fórceps. Referente à caracterização da amostra, verificou-se que $73,2 \%(n=180)$ das puérperas eram adultas e $26,8 \%(n=66)$ adolescentes. A maioria das mulheres era da cor branca $(64,2 \%)$, possuía companheiro $(88,2 \%)$, concluiu pelo menos o ensino fundamental completo $(82,5 \%)$, era primípara $(73,6 \%)$ e frequentou pelo menos seis consultas de pré-natal $(80,3 \%)$ (Tabela 1).

Quanto ao conhecimento sobre as condições de seu períneo após o parto, 96,3\% ( $n=237)$ das mulheres sabiam que tinham sido submetidas à episiotomia. Contudo, um total de $3,7 \%(n=9)$ desconhecia tal condição, este grupo não foi questionado sobre as variáveis a seguir, contudo, será apresentado juntamente às variáveis estudadas e permanecerá como parte dos resultados, sendo classificado como "desconheciam o estado do períneo". 
Tabela 1 - Caracterização das 246 mulheres segundo variáveis sociodemográficas e obstétricas. HCPA, Porto Alegre/RS, 2016.

\begin{tabular}{|c|c|c|}
\hline Variáveis & $\mathbf{n}$ & $\%$ \\
\hline \multicolumn{3}{|l|}{ Idade } \\
\hline $14-19$ anos & 66 & 26,8 \\
\hline 20-34 anos & 163 & 66,3 \\
\hline $35-41$ anos & 17 & 6,9 \\
\hline \multicolumn{3}{|l|}{ Cor } \\
\hline Branca & 158 & 64,2 \\
\hline Preta & 46 & 18,8 \\
\hline Parda/morena/mulata & 37 & 15,0 \\
\hline Amarela & 4 & 1,6 \\
\hline Indígena & 1 & 0,4 \\
\hline \multicolumn{3}{|l|}{ Situação marital } \\
\hline Possui companheiro & 217 & 88,2 \\
\hline Não possui companheiro & 29 & 11,8 \\
\hline \multicolumn{3}{|l|}{ Escolaridade } \\
\hline Ensino Fundamental Incompleto & 43 & 17,5 \\
\hline Ensino Fundamental Completo & 39 & 15,9 \\
\hline Ensino Médio Incompleto & 42 & 17,1 \\
\hline Ensino Médio Completo & 99 & 40,2 \\
\hline Ensino Superior Incompleto/Completo & 23 & 9,3 \\
\hline \multicolumn{3}{|l|}{ Paridade incluindo atual } \\
\hline Primípara & 181 & 73,6 \\
\hline Secundípara & 48 & 19,5 \\
\hline Tercípara/Multípara & 17 & 6,9 \\
\hline \multicolumn{3}{|l|}{ Número consultas pré-natal ${ }^{\star}$} \\
\hline$<6$ consultas & 48 & 19,7 \\
\hline$\geq 6$ consultas & 196 & 80,3 \\
\hline
\end{tabular}

Fonte: Jochims BO, Armellini CJ, Gouveia HG, 2016.

*Dado perdido: 2. 
Verificou-se que a maioria das mulheres, $77,2 \%(n=190)$, foi informada previamente à realização do procedimento que seria submetida à episiotomia, porém, $19,1 \%(n=47)$ negaram tal fato. Salienta-se que se avaliou o recebimento de informação no ambiente hospitalar (Figura 1).

Quando questionadas se receberam informação sobre o motivo de ter sido realizada a episiotomia, a maioria das mulheres, 65,0\% ( $n=160)$, respondeu sim, no entanto, ressalta-se que $2,8 \%(n=7)$ não soube informar tal motivo (Figura 1).

Figura 1 - Distribuição das mulheres submetidas à episiotomia $(n=246)$ segundo o recebimento de informação sobre o motivo da realização da episiotomia. HCPA, Porto Alegre - 2016.

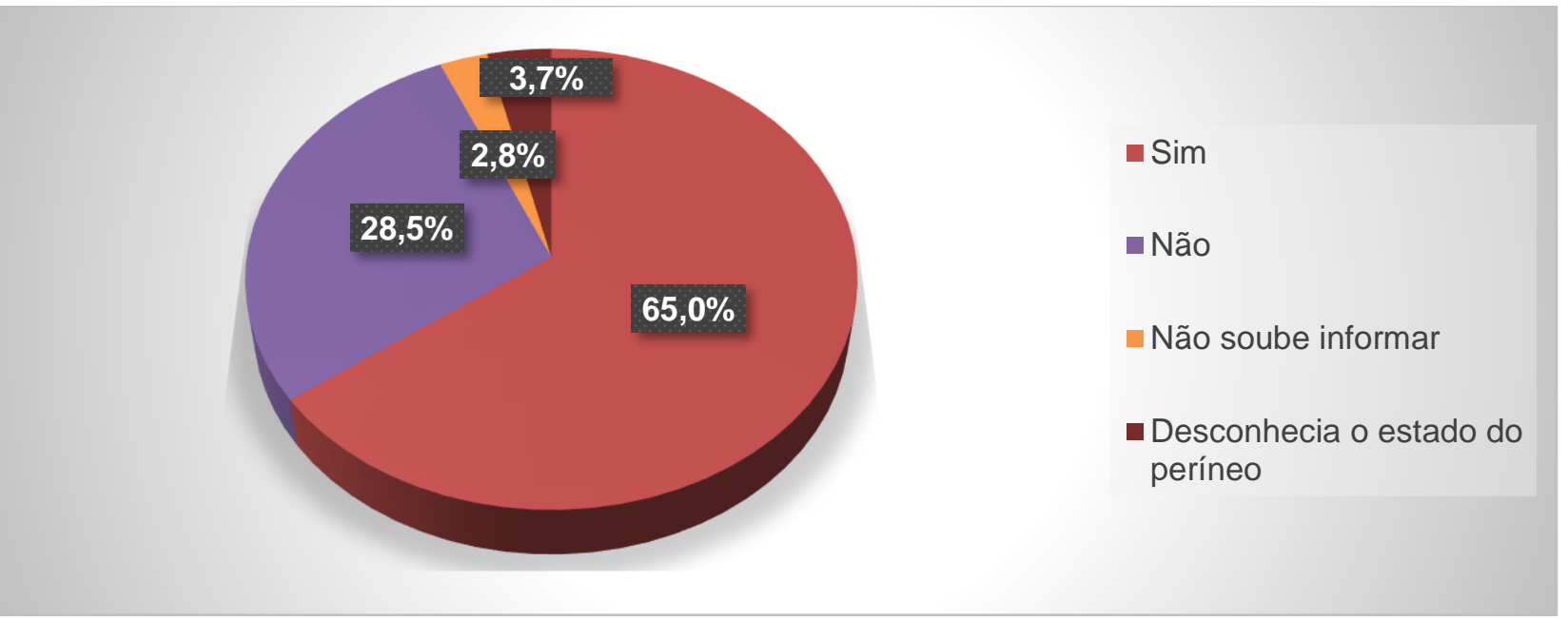

Fonte: Dados da pesquisa, 2016.

Entre as 160 mulheres que afirmaram ter recebido informação sobre o motivo da episiotomia, todas souberam informar o(s) motivo(s) (Tabela 2), sendo que o mais frequente, $75 \%$, foi "aumentar a passagem para o bebê".

Tabela 2 - Motivos de realização da episiotomia relatados pelas mulheres $(n=160)$. HCPA, Porto Alegre/RS, 2016.

\begin{tabular}{lrr}
\hline Motivos $^{*}$ & $\mathbf{n}$ & $\%$ \\
\hline Para aumentar a passagem para o bebê & 120 & 75,0 \\
Para nascer mais rápido & 24 & 15,0 \\
Bebê muito grande & 4 & 2,5 \\
Para não rasgar/lacerar o intestino & 4 & 2,5 \\
Para não rasgar/lacerar a vagina & 7 & 4,4 \\
Exaustão materna & 1 & 0,6 \\
Para retirar a placenta & 1 & 0,6 \\
Períneo era muito pequeno & 1 & 0,6 \\
Feto em posição occipto-púbica & 1 & 0,6 \\
Episiotomia prévia & 1 & 0,6 \\
\hline
\end{tabular}

Fonte: Jochims BO, Armellini CJ, Gouveia HG, 2016. *As puérperas podem ter referido mais de um motivo. 


\section{DISCUSSÃO}

Os dados desse estudo mostraram que entre as mulheres que foram submetidas à episiotomia, a maioria era adulta, resultado semelhante encontrado em estudo de Onofre BF (2014), realizado em 2012 na mesma instituição $(73,8 \%)$. Ainda em relação a faixa etária, encontrou-se um quarto de mulheres adolescentes, entretanto, em outro estudo, mais da metade das mulheres eram adolescentes (52,4\%) (PITANGUI ACR, et al., 2014). Há estudos que evidenciaram associação entre a prática da episiotomia e ser adolescente, sendo essas mais suscetíveis de sofrer a intervenção sob a justificativa de possuírem uma imaturidade anatômica como rigidez perineal e bacia em formação, além de imaturidade psicológica e mental (MELO JUNIOR EF, et al., 2006; OWA O, et al., 2015; PITANGUI ACR, et al., 2014).

Quando é avaliada a escolaridade das mulheres do estudo, percebe-se que a maioria concluiu pelo menos o ensino fundamental completo (82,5\%), dado semelhante a 78,4\% encontrado por Onofre BF (2014). Rasouli $\mathrm{M}$, et al. (2016) observaram que mulheres com nível universitário foram mais submetidas à episiotomia comparadas aquelas com nível escolar inferior, já Pitangui ACR, et al. (2014) não evidenciaram tal associação.

Verificou-se que um grande contingente das mulheres frequentou seis ou mais consultas durante o prénatal, número recomendado pelo Ministério da Saúde (BRASIL, 2012). Esse dado é semelhante ao resultado de $82,0 \%$ encontrado por Onofre BF (2014). Questiona-se a qualidade dessas consultas, visto que houve mulheres que não solicitaram o recebimento de informações sobre a episiotomia. Segundo Frigo J, et al. (2014), além do número mínimo de consultas preconizadas durante o pré-natal, é necessário que este seja efetivo, ou seja, baseado em orientações consistentes que visem o empoderamento da mulher, permitindo-a debater e decidir sobre intervenções que possam vir a ser realizadas em seu corpo, pois evidenciou-se que muitas são submetidas ao procedimento por falta de conhecimento. Sabe-se que mulheres que receberam educação continuada durante o pré-natal foram menos expostas a intervenções como a episiotomia (FIROUZBAKHT M, et al., 2015).

A maioria das mulheres desse estudo, era primípara, percentual próximo ao encontrado em outros estudos, como Rasouli M, et al. (2016) com 67,1\%, Onofre BF (2014) com 89,6\% e Hussein SAAA, et al. (2016) com 91\%. Dosler AJ, et al. (2017) e Owa O, et al. (2015) encontraram forte associação entre o uso da episiotomia e a primiparidade. Segundo Pitangui ACR, et al. (2014), as primíparas têm dez vezes mais chances de serem expostas ao procedimento, configurando-se, assim, a paridade um fator de risco para a episiotomia. Para Guimarães NNA, et al. (2018), a realização do procedimento em primíparas diminui o risco de laceração perineal, de relaxamento da musculatura pélvica posteriormente e de traumas à cabeça do feto.

A maior parte das mulheres deste estudo foi informada previamente de que seria submetida a episiotomia. Cabe informar que no hospital do estudo, no momento da admissão da mulher, é rotina ser apresentado por um integrante da equipe médica, um Termo de Consentimento Livre e Esclarecido onde está descrita a possibilidade de realização de alguns procedimentos durante o processo do parto e nascimento, entre eles, a episiotomia. Mas os resultados mostraram haver mulheres que referiram não terem sido informadas previamente quanto à realização da episiotomia. Verifica-se necessidade de melhorias quanto a aplicação desse Termo e a explicação fornecida à mulher a respeito deste Termo. Questiona-se sobre a compreensão da mulher acerca do que the foi explicado e se, no momento em que foi aplicado, a mulher estava apta emocionalmente para compreender e consentir tais procedimentos. Sugere-se que nesse momento a mulher esteja com seu acompanhante.

Comparando o percentual de mulheres, que relatou ter sido informada previamente sobre a episiotomia, com o resultado de Onofre BF (2014) constatou-se que, com o decorrer dos anos, houve um aumento de $20,4 \%$ de mulheres informadas previamente ao procedimento. Acredita-se que isso é decorrente do processo de qualificação da atenção ao parto na instituição. Mesmo assim, houve mulheres que não receberam tal informação, evidenciando que o desrespeito ao direito de informação ainda persiste.

Estudo de Rocha MJ e Grisi EP (2017) evidenciou que a maioria das mulheres foi informada sobre os procedimentos realizados no momento do parto, porém não possuía conhecimento suficiente para argumentar 
e compreendê-los de fato. Embora as mulheres sintam-se amedrontadas, constrangidas, desconfortáveis, impotentes e vulneráveis à dor perante a episiotomia, ainda há a crença de que esse procedimento é normal e necessário para a mãe e o bebê devido à falta de informação e conhecimento da fisiologia do parto (CARVALHO PD, et al., 2015; POMPEU KC, et al., 2017). A falta de questionamento das mulheres acerca deste procedimento pode estar relacionada ao referencial que elas possuem do médico, vendo-o como agente principal e indispensável para a realização do nascimento, depositando confiança na tomada de decisões médicas, tendo estas como necessárias, estabelecendo assim, uma relação de poder do profissional de saúde sobre a mulher (POMPEU KC, et al., 2017; VILLELA JP, et al., 2016).

Quanto ao recebimento de informação sobre o motivo para a realização da episiotomia, a maioria das puérperas afirmou ter sido informada. Para Odent M (2002), durante o trabalho de parto, a parte cerebral primitiva da mulher está mais ativa, enquanto o neocórtex, a parte mais racional do cérebro, está com atividade reduzida. A mulher é exposta a muitos sentimentos em um curto período de tempo e, devido a isso, pode não lembrar ou fixar as informações fornecidas a ela nesse momento. Por isso, é importante identificar o momento oportuno para realização de orientações, podendo iniciar no pré-natal, para que haja tempo de entendimento da mulher e de seu companheiro sobre as questões que envolverão o processo de parturição.

O motivo prevalente de ter sido submetida à episiotomia, referido pelas mulheres do presente estudo foi "para aumentar a passagem para o bebê", já no estudo anterior (ONOFRE BF, 2014) foi "ajudar o bebê a nascer" $(68,8 \%)$, esses motivos podem ser considerados semelhantes levando em consideração o desfecho esperado para mulher. $\mathrm{O}$ atual estudo encontrou percentuais inferiores comparados a outros motivos encontrado por Onofre BF (2014) como "bebê grande" (14,0\%), "falta de força/exaustão materna" $(3,2 \%)$ e "episiotomia prévia" $(1,1 \%)$. Em contrapartida, encontrou-se um percentual superior aos 2,1\% encontrados por Onofre BF (2014) de episiotomia realizada pelo motivo "não rasgar/não lacerar" no qual podem estar inclusos a vagina e/ou o intestino. Apesar de Jiang $\mathrm{H}$, et al. (2017) comprovarem que a episiotomia não exerce efeito protetor sobre a condição fetal, ainda há na literatura indicações da episiotomia em casos de condição fetal não tranquilizadora, macrossomia fetal, distócia de ombros, apresentação pélvica, algumas variedades posteriores da apresentação fetal, condições maternas que impeçam realização de puxos dirigidos, corpo perineal inferior a três centímetros e possibilidade de realização de parto instrumentalizado (MARTINSCOSTA SH, et al., 2017).

Questiona-se, assim, a qualidade da assistência recebida quanto à informação sobre as indicações da episiotomia, ou seja, se essa informação não foi fornecida ou ocorreu em momento inadequado, dificultando a compreensão da mulher, e ao direito de a mulher opinar sobre os procedimentos realizados em seu corpo. Sabe-se que não há critérios exatos para a realização da episiotomia, porém a recomendação ideal deve garantir menores taxas de complicações no nascimento (SAGI-DAIN L e SAGI S, 2015). Algumas justificativas evidenciadas pelas mulheres do atual estudo, como períneo muito pequeno e exaustão materna, remetem a incompetência da mulher e de sua fisiologia, tratando-a como ser passivo na reprodução, pois infere que ela é incapaz de parir sozinha, necessitando de alguém portador de conhecimento para ajudar no nascimento de seu filho (PRIETO LNT, 2015).

Em 2014 foi implementado protocolo que visava o uso restritivo da episiotomia no hospital deste estudo, o que pode ter contribuído para a redução de $17,1 \%$ do índice de episiotomia se comparados aos índices encontrados nos estudos com coletas na mesma instituição nos anos de 2012 (ONOFRE BF, 2014) e de 2016, estudo atual.

Neste estudo, quando as puérperas foram questionadas sobre as condições do seu períneo, quase a totalidade referiu ter este conhecimento. Comparando estes achados com os de 2012 na mesma instituição, percebeu-se que houve um aumento de $7,2 \%$ de mulheres que conheciam as condições do seu períneo, fato que pode estar relacionado ao recebimento de informação sobre a episiotomia durante o pré-natal, e/ou até mesmo, imediatamente antes, durante ou após a realização do procedimento. Por outro lado, houve mulheres que desconheciam ter sido submetidas à episiotomia, mesmo sendo este um procedimento cirúrgico para o qual é necessário consentimento prévio, conforme estabelecido pelo Código de Ética Médica em seu Artigo $22^{\circ}$ e $24^{\circ}$, ficando vedado ao médico não obter consentimento do paciente e não garanti-lo direito de decidir 
sobre si mesmo e também assegurado pelo Código Penal em seu Artigo 129ํㅜ, que considera lesão corporal de natureza grave realizar atos que resulte em debilidade permanente de membro ou função e aceleração do parto (BRASIL, 1940; CONSELHO FEDERAL DE MEDICINA, 2009). O desconhecimento das mulheres sobre as condições de seu períneo pós-parto remete a discussão sobre o desrespeito da sua individualidade e dos seus direitos, e sobre a precariedade na oferta de informação (VILLELA JP, et al., 2016).

Esse estudo tem como limite a escassez de pesquisas com abordagem quantitativa sobre 0 tema. Entretanto, há estudos qualitativos que apontam a desconsideração dos profissionais para com as mulheres, submetendo-as à episiotomia sem a informação ou o consentimento prévio acerca do procedimento, sem considerar que a realização de procedimentos sem o consentimento da mulher pode gerar sequelas psicológicas ou físicas, além de aumentarem as dores e gerarem um sentimento de participação insatisfatória no trabalho de parto (BOLSONI AC e COELHO JA, 2016; CARVALHO PB, et al., 2015; DENGO VAR, et al., 2016; FRIGO J, et al., 2014; GARRETT CA, et al., 2016; MEDEIROS NCM, et al., 2016).

A falta de conhecimento das mulheres sobre o motivo de ter sido submetida à episiotomia é evidenciada nos estudos de Bolsoni AC e Coelho JA (2016), onde a maioria delas não sabia por que sofreu a intervenção, e de Frigo J, et al. (2014), onde para 50\% ( $n=14)$ das mulheres o profissional não comentou ou justificou a necessidade do procedimento. As mulheres do presente estudo relataram outros motivos para justificar a realização da episiotomia, semelhantes a motivos citados em estudos qualitativos onde as mulheres relataram terem sido submetidas à episiotomia sob o pretexto de facilitar o parto, o bebê ser muito grande, o períneo ser muito pequeno, auxiliar o bebê nascer, ter episiotomia prévia ou ser primípara (CARVALHO PB, et al., 2015; DENGO VAR, et al., 2016; FRIGO J, et al., 2014).

Estudos apontam algumas situações relacionadas a realização da episiotomia, como o profissional tomar para si essa decisão, seja pela impaciência em esperar o nascimento do bebê sob o pretexto da superlotação das salas de parto, pela incapacidade de perceber que a vagina é um órgão elástico e capaz de se distender ou intenção de abreviar o período expulsivo (CARVALHO PD, et al., 2015; DINIZ CSJ, 2012; RIESCO MLG, 2014; SCHANTZ C, et al., 2015;). Há de se lembrar que a episiotomia é algo que perpassa as gerações, sendo ensinada na academia, e consequentemente, impactando o atendimento à mulher (BRASIL, 2001).

A comunicação entre o profissional e a mulher mostra-se como um fator relevante que contribui para a satisfação da mesma com o parto, pois a coloca como parte do processo (MARTINS ACM, 2017). Para modificar o cenário atual da obstetrícia é necessário que o sistema de saúde se responsabilize pelo atendimento oferecido às mulheres durante a gestação e o parto, por meio da implementação de políticas que esclareçam os direitos e deveres do cidadão, e que os profissionais de saúde recebam treinamento e apoio para assegurar o atendimento digno à mulher (ORGANIZAÇÃO MUNDIAL DA SAÚDE, 2014). Tais modificações contribuirão para que os profissionais repensem sua conduta acerca de práticas rotineiras e disseminem informações atualizadas, visando o empoderamento da mulher, permitindo-a dialogar sobre as intervenções e rejeitá-las quando necessário (SANTOS ADR, et al., 2017). Para que a eliminação de abusos e a diminuição da violência obstétrica sejam viáveis, é necessária a criação de processos inclusivos, onde profissionais de saúde, gestores, mulheres e educadores trabalhem juntos para tal fim (ORGANIZAÇÃO MUNDIAL DA SAÚDE, 2014).

Como limitações deste estudo, não foi questionado sobre qual profissional informou o motivo da realização da episiotomia, visto que tal motivo deveria ser informado pelo profissional que a executou, nem em qual momento isso ocorreu, ou seja, antes, durante ou após sua realização. Não foi possível avaliar se o relato das puérperas sobre o motivo pelo qual foram submetidas à episiotomia coincide com a indicação deste procedimento no documento de descrição do parto, pois no período da coleta não havia um campo para esse registro. Posteriormente, tal documento foi modificado e inserido campo obrigatório para registro da indicação da episiotomia.

Sugere-se a realização de novos estudos de abordagem quantitativa sobre a temática deste estudo a fim de conhecer a situação em outros contextos para que sejam pensadas medidas de modificação do cenário atual e seja possível analisar se tais modificações foram efetivas. 


\section{CONCLUSÃO}

Os resultados evidenciaram que a maioria das mulheres conhecia as condições de seu períneo, foi informada que seria submetida à episiotomia e recebeu informação sobre o motivo desse procedimento. Constatou-se que, apesar de ser um direito das mulheres de receber informações e esclarecimentos sobre quaisquer procedimentos que venham a ser realizados no seu corpo, ainda houve aquelas que não foram contempladas com essas informações. Ressalta-se assim, a necessidade de estabelecer uma comunicação efetiva e de confiança entre a equipe de saúde e a mulher, mantendo-a informada sobre os possíveis procedimentos e esclarecida quanto as suas dúvidas, visando o respeito, a autonomia e o empoderamento sobre o seu parto.

\section{REFERÊNCIAS}

1. ANDRADE BP, AGGIO CM. Violência obstétrica: a dor que cala. Simpósio Gênero e Saúde Pública, $2014 ; 3$.

2. ANDRADE MAC, LIMA JBMC. O modelo obstétrico e neonatal que defendemos e com o qual trabalhamos. Humanização do parto e do nascimento. Brasília: Ministério da Saúde, 2014; 467.

3. BOLSONI AC, COELHO JA. Episiotomia no puerpério: percepção das mulheres. Revista de Saúde Pública do Paraná, 2016; 17(2): 199-205.

4. BRASIL. Atenção ao pré-natal de baixo risco. Brasília, DF: Ministério da Saúde, 2012; 320 p.

5. BRASIL. Parto, aborto e puerpério: assistência humanizada à mulher. Brasília, DF: Ministério da Saúde, $2001 ; 202$ p.

6. BRASIL. Decreto Lei no 2.848, de 07 de dezembro de 1940. Código Penal. Diário Oficial da União, Poder Executivo, Brasília, DF, 31 dez. 1940. Seção 1, p. 23911.

7. CARVALHO PD, et al. Percepção de puérperas quanto ao procedimento da episiotomia. Journal of the Health Sciences Institute, 2015; 33(3): 228-234.

8. CONSELHO FEDERAL DE MEDICINA. Resolução № 1.931, de 17 de setembro de 2009. Código de Ética Médica. Brasília, DF: CFM, 2009.

9. DENGO VAR, et al. Puerperal women's perceptions about episiotomy. Cogitare Enfermagem, 2016; 21 (3): 1-8.

10. DINIZ CSG. Violência obstétrica: "parirás com dor". [Brasília, DF]: Parto do Princípio, 2012; 188 p.

11. DOSLER AJ, et al. Incidence of episiotomy in Slovenia: The story behind the numbers. Nursing and Health Sciences, 2017; 19(3): 351-357.

12. FIROUZBAKHT M, et al. The effectiveness of prenatal intervention on pain and anxiety during the process of childbirth-northern Iran: clinical trial study. Annals of Medical and Health Sciences Research, 2015; 5(5): 348-352.

13. FRIGO J, et al. Episiotomia: (des)conhecimento sobre o procedimento sob a ótica da mulher. Brazilian Journal of Surgery and Clinical Research, 2014; 6(2): 5-10.

14. GARRETT CA, OSELAME GB, NEVES EB. O uso da episiotomia no sistema único de saúde brasileiro: a percepção das parturientes. Saúde e Pesquisa, 2016; 9(3): 453-459.

15. GUIMARÃES NNA, et al. Análise de fatores associados à prática da episiotomia. Revista de Enfermagem UFPE on line, 2018; 12(4): 1046-1053.

16. HUSSEIN SAAA, et al. The barriers and facilitators to evidence-based episiotomy practice in Jordan. Women Birth, 2016; 29(4): 321-329.

17. JIANG H, et al. Selective versus routine use of episiotomy for vaginal birth. Cochrane Database of Systematic Reviews, 2017; 2: 1-50.

18. MARTINS ACM. Fatores associados à maior satisfação das mulheres com o atendimento ao parto em maternidades do Sul do Brasil. Dissertação (Mestrado em Epidemiologia). Universidade Federal do Rio Grande do Sul, 2017, $58 \mathrm{p}$.

19. MARTINS-COSTA SH, et al. Rotinas em obstetrícia. 7. ed. Porto Alegre: Artmed, 2017; 894 p.

20. MEDEIROS NCM, et al. Violência obstétrica: percepções acerca do parto normal. Temas em Saúde, 2016; 16(3): 503-528.

21. MELO JUNIOR EF, et al. Fatores associados à realização seletiva de episiotomia em hospital universitário. Revista de Ciências Médicas, 2006; 15(2): 95-101.

22. ODENT M. A cientificação do amor. 2. ed. Florianópolis: Saint Germain, 2002; 139 p.

23. ONOFRE BF. Mulheres submetidas à episiotomia e as informações quanto à realização deste procedimento. TCC (Bacharelado em Enfermagem) - Escola de Enfermagem. Universidade Federal do Rio Grande do Sul, Porto Alegre, 2014, 49 p. 
24. ORGANIZAÇÃO MUNDIAL DA SAÚDE. Prevenção e eliminação de abusos, desrespeito e maus-tratos durante o parto em instituições de saúde. Genebra: Organização Mundial da Saúde, 2014, 4 p.

25. OWA O, ENIOWO A, ILESANMI O. Factors associated with episiotomy among parturients delivering in a tertiary care centre in Nigeria. International Journal of Research in Medical Sciences, 2015; 3(4): 836-840.

26. PITANGUI ACR, et al. Ocorrência e fatores associados à prática de episiotomia. Revista de Enfermagem UFPE on line, 2014; 2(8): 257-263.

27. POMPEU KC, et al. Prática da episiotomia no parto: desafios para a enfermagem. Revista de Enfermagem do Centro-Oeste Mineiro, 2017; 7(e2242).

28. PRIETO LNT. A episiotomia de rotina é uma prática baseada em evidência? Uma revisão integrativa de literatura. Monografia (Bacharelado em Enfermagem). Universidade de Brasília, 2015, 18 p.

29. RASOULI M, et al. Prevalence and factors associated with episiotomy in Shahroud City, Northeast of Iran. International Journal of Women's Health and Reproduction Sciences, 2016; 4(3): 125-129.

30. RIESCO MLG. Nascer no Brasil: uma questão de hierarquia das intervenções no parto. Cadernos de Saúde Pública, 2014; 30(supl.): S35-S36.

31. ROCHA MJ, GRISI EP. Violência obstétrica e suas influências na vida das mulheres que vivenciaram essa realidade. Id on Line, Revista Multidisciplinar e de Psicologia, 2017:11(38); 623-635.

32. SAGI-DAIN L, SAGI S. The correct episiotomy: does it exist? A cross-sectional survey of four public Israeli hospitals and review of the literature. International Urogynecology Journal, 2015; 26(8): 1213-1219.

33. SANTOS ADR, et al. Episiotomia: a dor de um parto. Cadernos de Graduação, 2017; 4(1): 131-138.

34. SCHANTZ C, et al. Reasons for routine episiotomy: a mixed-methods study in a large maternity hospital in Phnom Penh, Cambodia. Reproductive Health Matters, 2015; 23(45): 68-77.

35. VOGT SE, et al. Comparação de modelos de assistência ao parto em hospitais públicos. Revista de Saúde Pública, 2014; 48(2): 304-313.

36. WORLD HEALTH ORGANIZATION. Who recommendations: Intrapartum care for a positive childbirth experience. Geneva: World Health Organization, 2018, 210 p. 